\title{
Optimization of Grafted Fibrous Polymer as a Solid Basic Catalyst for Biodiesel Fuel Production
}

\author{
Yuji Ueki*, Seiichi Saiki, Takuya Shibata, Hiroyuki Hoshina, Noboru Kasai, Noriaki Seko \\ Environment and Industrial Materials Research Division, Quantum Beam Science Center, Sector of Nuclear \\ Science Research, Japan Atomic Energy Agency, Takasaki, Japan \\ Email: ${ }^{*}$ ueki.yuji@jaea.go.jp
}

Received 3 April 2014; revised 8 May 2014; accepted 16 May 2014

Copyright @ 2014 by authors and Scientific Research Publishing Inc.

This work is licensed under the Creative Commons Attribution International License (CC BY). http://creativecommons.org/licenses/by/4.0/

c) (i) Open Access

\section{Abstract}

Grafted fibrous polymer with quaternary amine groups could function as a highly-efficient catalyst for biodiesel fuel (BDF) production. In this study, the optimization of grafted fibrous polymer (catalyst) and transesterification conditions for the effective BDF production was attempted through a batch-wise transesterification of triglyceride (TG) with ethanol (EtOH) in the presence of a cosolvent. Trimethylamine was the optimal quaternary amine group for the grafted fibrous catalyst. The optimal degree of grafting of the grafted fibrous catalyst was greater than $170 \%$. The optimal transesterification conditions were as follows: The optimal molar quantity of quaternary amine groups, transesterification temperature, molar ratio of TG and EtOH, and primary alkyl alcohol were $0.8 \mathrm{mmol}, 80^{\circ} \mathrm{C}, 1: 200$, and 1-pentanol, respectively. The grafted fibrous catalyst could be applied to BDF production using natural oils. Furthermore, the grafted fibrous catalyst could be used repeatedly after regeneration involving three sequential processes, i.e., organic acid, alkali, and alcohol treatments, without any significant loss of catalytic activity.

\section{Keywords}

Biodiesel Fuel, Triglyceride Transesterification, Radiation-Induced Graft Polymerization, Grafted Polymer, Heterogeneous Basic Catalysis

\section{Introduction}

The global energy consumption has increased every year and has more than doubled between 1970 and 2008

*Corresponding author. 
(1970: 207 quadrillion British thermal units (Btu); 2008: 505 quadrillion Btu); experts predict that it will increase by another $53 \%$ by 2035 to become 770 quadrillion Btu [1] [2]. This growing global demand for energy will likely lead to the depletion of fossil fuels, unprecedented emissions of air pollutants and greenhouse gases, high energy-resource prices, and regional conflicts due to uneven distribution of fossil fuels. Therefore, to avoid and overcome these problems, many researchers worldwide are working on the development of methods to harvest energy from renewable sources such as solar, wind, hydraulic, geothermal, and biomass, together with innovative practical technologies. Among these, biomass energy is garnering attention because it has a high energy density and requires relatively facile handling and storage. Currently, studies on the production of biomethane from cellulose and organic waste [3] [4], production of bioethanol from starch and nonfood crops [5] [6], conversion of vegetable oils, animal fats, and waste oils into biodiesel fuel (BDF) [7]-[15], and production of biofuels from algae [16] [17] are in progress.

BDF is defined as the monoalkyl esters of long-chain fatty acids (carbon chain lengths of 12 - 24), and is generally produced by transesterification of triglyceride (TG), which is the main constituent of vegetable oils and animal fats, with short-chain primary alcohols such as methanol or ethanol (EtOH). BDF has a number of important advantages: It is renewable, environmentally friendly, easily biodegradable, and compatible with current fuel infrastructure, and its production process, transport, and storage are very straightforward. Additionally, BDF can be used in compression-ignition engines instead of petroleum diesel with little or no modifications to the engine components. For these reasons, BDF has attracted significant attention as an alternative fossil fuel. BDF production can be classified into several methods according to the type of catalyst: homogeneous catalyst (alkaline and acid), heterogeneous catalyst (metal oxides, carbonates, zeolites, heteropoly acids, functionalized zirconia or silica, ion-exchange resins, hydrotalcites and alkaline salts) and enzymatic (lipases) catalysts and a noncatalytic supercritical method [7]-[15]. Some methods for the industrial production of BDF from oil and fat have already been developed; currently, an alkali catalyst method using a homogeneous catalyst such as $\mathrm{NaOH}$ or $\mathrm{KOH}$ is predominantly used because of its relatively fast reaction rates. However, this method has some disadvantages: The alkali catalyst corrodes equipment, the reaction requires a large amount of water during the neutralization and washing processes, undesirable byproducts are produced by a saponification side reaction, and it is difficult to separate the homogenized catalyst from the reaction mixture. These problems increase the BDF production costs.

To solve or minimize these problems, authors proposed the use of grafted fibrous polymer as a solid basic catalyst [18]. Grafted fibrous polymer (grafted fibrous catalyst) was synthesized by radiation-induced graft polymerization which could impart a desired functional group into pre-existing polymeric materials (i.e., trunk polymer) without altering their inherent properties, and the grafted fibrous polymer was used as adsorbents for environmental reclamation and obtaining resources [19]-[25]. In particular, when nonwoven fabric having both large specific surface area and high contact efficiency is selected as a trunk polymer for grafting, the BDF production speed (i.e., transesterification speed) of the grafted fibrous catalyst was more than twice that of commercial granular anion exchange resin, and the grafted fibrous catalyst could efficiently produce BDF within a shorter period of time [18]. This difference in the transesterification speed of the grafted fibrous catalyst and anion exchange resin was mostly attributed to their shape differences: The porous anion exchange resin had numerous micropores that increase the surface area. Thus, most of the reaction sites (i.e., functional groups) of the porous resin were located within its micropores, and hence the reactants were transported to the functional groups by diffusion (i.e., concentration gradient). Therefore, diffusional mass-transfer was the rate-determining factor for BDF production. In contrast, in the grafted fibrous catalyst, the functional groups were immobilized onto the graft chains, and the reactants were easily and immediately transported to the functional groups by convective flow of the reaction mixture. Therefore, the diffusional mass-transfer resistance of the reactants to the functional groups could be neglected. Additionally, the catalyst had further advantages: it was less corrosive than homogeneous alkali catalysts, did not produce soap, and was easily recovered from the BDF, which negated the need for neutralization and washing. For these advantages, authors think that the grafted fibrous catalyst will contribute to simplify and streamline the BDF production process, reduce the BDF production costs, reduce the waste product, and establish the large-scale BDF production process.

In our previous paper, we found that the grafted fibrous catalyst could function as a highly-efficient catalyst for BDF production [18]. The objective of this study is to characterize the catalytic properties of the grafted fibrous catalyst and determine the optimal BDF production conditions using the grafted fibrous catalyst. First, we demonstrated the effectiveness of type of immobilized quaternary amine groups onto the grafted fibrous cat- 
alyst, from both perspectives of the ease of amination and the catalytic performance. Second, the effects of the catalyst and transesterification conditions on the catalytic activity, transesterification speed, and production yield of BDF were elucidated by batch-wise transesterification of TG with EtOH in the presence of a cosolvent. Additionally, the effect of the type of natural oil on the transesterification yield and speed was investigated. Finally, the recyclability of the grafted fibrous catalyst and regeneration of the deactivated grafted fibrous catalyst were evaluated.

\section{Experimental}

\subsection{Materials}

Polyethylene-coated polypropylene (PE/PP) nonwoven fabric was supplied by Kurashiki Textile Manufacturing Co., Ltd. (Osaka, Japan) and used as a trunk polymer for BDF catalysts. The average fiber diameter of the PE/ PP nonwoven fabric was $13 \mu \mathrm{m}$. 4-Chloromethylstyrene (CMS; purity > 95\%) was purchased from AGC Seimi Chemical Co., Ltd. (Kanagawa, Japan). Polyoxyethylene (20) sorbitan monolaurate (Tween 20), which was used as a nonionic surfactant for preparing the monomer emulsion, was obtained from Kanto Chemical Co., Inc. (Tokyo, Japan), as were trimethylamine (TMA), triethylamine (TEA), tri-n-propylamine (TPA), tri-n-butylamine (TBA), triolein (purity $>60 \%$ ), and oleic acid (purity $>80 \%$ ). Sodium hydroxide, methanol, EtOH, 1-propanol, 2-propanol, 1-butanol, 1-pentanol, 1-hexanol, 1-octanol, 1-decanol, 1-dodecanol, acetonitrile, hexane, decane, linseed oil, beef tallow, and citric acid were supplied by Wako Pure Chemical Industries, Ltd. (Osaka, Japan). Rapeseed oil (51\% erucic acid) and safflower oil (food-grade, 70\% - 80\% linoleic acid) were purchased from MP Biomedicals, LLC. (CA, USA). Palm oil (32\% - 47\% palmitic acid, 34\% - 44\% oleic acid) was obtained from Spectrum Chemical Mfg. Corp. (NJ, USA). EtOH, 2-propanol, acetonitrile, and hexane were of HPLC grade and all other chemicals were of reagent grade unless otherwise stated. Deionized water obtained from a Milli-Q deionization system (Nihon Millipore K.K., Tokyo, Japan) was used directly for preparing the monomer emulsions and aqueous solutions.

\subsection{Synthesis of Grafted Fibrous Catalyst for Biodiesel Fuel Production}

Grafted fibrous catalyst for BDF production was synthesized by radiation-induced emulsion grafting technique, as published previously [18]. PE/PP nonwoven fabric was irradiated with electron beam (100 kGy). Then, the irradiated PE/PP nonwoven fabric reacted with a deaerated monomer emulsion, which was composed of 3 wt\% CMS, $0.3 \mathrm{wt} \%$ tween 20 and $96.7 \mathrm{wt} \%$ deionized water, in a glass ampoule and was kept in water bath at $40^{\circ} \mathrm{C}$. Afterward, the CMS-grafted nonwoven fabric was recovered from the emulsion and washed repeatedly with water and then methanol to remove residual monomers and homopolymers. The amount of CMS grafted onto the PE/PP nonwoven fabric was evaluated by the degree of grafting ( $\mathrm{Dg}[\%]$ ), which was calculated using the following equation:

$$
D g[\%]=100 \times\left(W_{1}-W_{0}\right) / W_{0},
$$

where $W_{0}$ and $W_{1}$ are the dry weights of the PE/PP nonwoven fabrics before and after grafting, respectively.

To introduce quaternary amine groups into the CMS-graft chains of CMS-grafted nonwoven fabric (catalyst precursor), the catalyst precursor was treated with 0.25 and $2.5 \mathrm{M}$ amine-ethanol solutions at $60^{\circ} \mathrm{C}$ for $24 \mathrm{~h}$. To eliminate residual amine reagents, the aminated catalyst precursor was then washed with deionized water until it was neutral. The resultant aminated catalyst precursor is referred to as the grafted fibrous catalyst. The quaternary amine-group densities of the grafted fibrous catalysts were estimated by an analysis of their nitrogen content using an elemental analyzer (model: 2400 Series II CHNS/O elemental analyzer, PerkinElmer, Inc., MA, USA). The quaternary amine-group density of the grafted fibrous catalyst and degree of amination ( $\mathrm{Da}[\%])$ of the CMS-graft chain are defined as follows:

Quaternary amine-group density $[\mathrm{mmol}$-amine/g-catalyst $]=(N / 100) / 14 \times 1000$, and

$$
D a[\%]=100 \times\left[152.6 \times\left(W_{2}-W_{0}\right) /\left(W_{1}-W_{0}\right)-152.6\right] / M_{\text {Amine }},
$$

where $N, W_{2}$, and $M_{\text {Amine }}$ are the measured nitrogen content (\%) of the grafted fibrous catalyst, weight of the grafted fibrous catalyst, and molecular weight of the introduced amine compound, respectively. The molecular weights of TMA, TEA, TPA, and TBA are 59.11, 101.19, 143.27 and $185.35 \mathrm{~g} / \mathrm{mol}$, respectively, while the val- 
ues 14 and 152.6 refer to the molecular masses of $\mathrm{N}$ and CMS, respectively.

\subsection{Transesterification Procedure with Grafted Fibrous Catalyst}

The catalytic performance of the grafted fibrous catalyst was evaluated by the transesterification of TG and alcohols under the following standard conditions, as published previously [18].

The transesterification tests were performed in a batch reactor equipped with a magnetic stirrer. The reactor was initially filled with $1.4 \mathrm{~g}(1.6 \mathrm{mmol})$ of triolein as a TG and $3.6 \mathrm{~g}(78 \mathrm{mmol})$ of EtOH as an alcohol, followed by the addition of $5.0 \mathrm{~g}$ of decane as a cosolvent in order to obtain a homogeneous phase. After stirring for $10 \mathrm{~min}$, approximately $0.2 \mathrm{~g}$ of the pretreated grafted fibrous catalyst was immersed in the homogenous reaction solution, and the resultant mixture was heated to the reaction temperature (standard: $50^{\circ} \mathrm{C}$ ) while stirring at $600 \mathrm{rpm}$. The reaction time is set at zero when the grafted fibrous catalyst was added to the reaction solution. In a typical test, the molar ratio of TG to EtOH was 1:50, and the $D g$, type of quaternary amine group, and amine group density of the grafted fibrous catalyst were $~ 300 \%$, TMA, and $3.6 \mathrm{mmol}$-amine/g-catalyst, respectively. The molar ratio of TG to quaternary amine groups in the reaction mixture was fixed at 2:1, i.e., $0.8 \mathrm{mmol}$ of quaternary amine group of the grafted fibrous catalyst was in the reaction mixture per test. At pertinent intervals, $0.1 \mathrm{~mL}$ aliquots were withdrawn from the reaction solution, quenched to room temperature, and diluted to 10 times the volume with 2-propanol/hexane $(5: 4, \mathrm{v} / \mathrm{v})$ for compositional analysis. The effect of the molar ratio of TG to quaternary amine group (16:1 - 2:1), type of quaternary amine group, reaction temperature $\left(20^{\circ} \mathrm{C}-\right.$ $\left.80^{\circ} \mathrm{C}\right)$, molar ratio of TG to EtOH (1:3 - 1:500), type of alcohol, and reaction time on the conversion ratio of TG to biodiesel fuel were investigated in detail.

\subsection{Compositional Analysis}

The compositions of the collected samples were analyzed using high-performance liquid chromatography (HPLC), according to the separation conditions described by Holčapek et al. [26]. The HPLC system (Shimadzu, Kyoto, Japan) consisted of a pump (model: LC-20AD) with a quaternary gradient system, degasser (model: DGU-20A5), system controller (model: CBM-20Alite), sample injector (model: 7725i; Rheodyne, CA, USA) with a $1.0 \mu \mathrm{L}$ sample reservoir, column oven (model: CTO-20AC), and UV-Vis detector (model: SPD-20A). System control and data processing were carried out using Shimadzu LC solution software. A reversed-phase C18 column (model: L-column ODS, column size: $2.1 \mathrm{~mm}$ i.d. $\times 150 \mathrm{~mm}$ long, particle diameter: $5 \mu \mathrm{m}$, Chemicals Evaluation and Research Institute, Tokyo, Japan) was used for separation. The compositional analysis was conducted with a quaternary mobile phase consisting of deionized water (solvent A), acetonitrile (solvent B), 2-propanol (solvent $\mathrm{C}$ ), and hexane (solvent $\mathrm{D}$ ). The quaternary gradient elution program was as follows: $30 \% \mathrm{~A}$ $+70 \% \mathrm{~B}$ at $0 \mathrm{~min}, 100 \% \mathrm{~B}$ at $5 \mathrm{~min}, 50 \% \mathrm{~B}+27.8 \% \mathrm{C}+22.2 \% \mathrm{D}$ at $10 \mathrm{~min}$, and isocratic elution with $50 \% \mathrm{~B}$ $+27.8 \% \mathrm{C}+22.2 \% \mathrm{D}$ for the last $5 \mathrm{~min}$. The flow rate was set at $1.0 \mathrm{~mL} / \mathrm{min}$, the effluent was monitored at 205 $\mathrm{nm}$, and the column temperature was maintained at $40^{\circ} \mathrm{C}$. The conversion ratio of TG to BDF was calculated from the rate of change of the total HPLC peak areas for TG before and after transesterification.

\section{Results and Discussion}

\subsection{Effect of Type of Quaternary Amine Groups on Catalytic Performance}

To produce BDF using grafted fibrous catalyst, $\mathrm{OH}^{-}$ions, which were chemically immobilized on the grafted fibrous catalyst, are the actual catalytic elements, while the polymer matrix acts as a scaffold to immobilize these ions. Although $\mathrm{OH}^{-}$ions can be easily immobilized onto the grafted fibrous catalyst by interionic interaction with the cationic functional groups such as a quaternary amine group, it is considered that the type of quaternary amine group has possibility to have a significant influence on the catalytic performance. In order to select the best quaternary amine group of grafted fibrous catalyst, we discussed the effects of four types of amines with different alkyl chain lengths, such as TMA, TEA, TPA, and TBA, from both perspectives of the reactivity with CMS-grafted chains and the catalytic performance.

A CMS-grafted nonwoven fabric with $170 \% D g$ was used as the catalyst precursor, and each amine concentration was fixed at $0.25 \mathrm{M}$. As expected, the quaternary amine-group density increased with increasing reaction time, and TMA, which has the shortest alkyl chain length, exhibited the highest reactivity toward the CMS-graft chains. As shown in Figure 1, amination with 0.25 M TMA began immediately and completed within $1 \mathrm{~h}$; the 
quaternary amine-group density and $\mathrm{Da}$ reached $3.0 \mathrm{mmol}-\mathrm{TMA} / \mathrm{g}$-catalyst and 93\%, respectively. However, other amine reagents with longer alkyl chain lengths, such as TEA, TPA, and TBA, were less reactive because of the increased steric hindrance of the amine compounds. Amination of the CMS-graft chains did not completely finish even after $24 \mathrm{~h}$ of amination with $0.25 \mathrm{M}$ amine solution; the $D a$ for the products of the reactions with TEA, TPA, and TBA were $81 \%, 63 \%$, and $65 \%$, respectively. To enhance these values, the CMS-grafted nonwoven fabric was treated with 2.5 M TEA-, TPA-, and TBA-EtOH solutions. After $24 \mathrm{~h}$ of amination with 2.5 M TEA, TPA, and TBA, $D a$ values of $91 \%, 85 \%$, and $88 \%$, respectively, were achieved. These values are comparable to that for TMA. However, longer alkyl chain lengths of the amine compounds led to reduced quaternary amine-group densities per gram of grafted fibrous catalysts, even when the $D a$ values for each amine were comparable to that of TMA. As denoted in Figure 1, the quaternary amine-group density for each amine reached $2.6 \mathrm{mmol}-\mathrm{TEA} / \mathrm{g}$-catalyst, $2.2 \mathrm{mmol}$-TPA/g-catalyst, and $2.0 \mathrm{mmol}-\mathrm{TBA} / \mathrm{g}$-catalyst after $24 \mathrm{~h}$ of amination. The effect of the $D g$ of the CMS-grafted nonwoven fabric on amination was also investigated, and it was found that the $D g$ value did not significantly influence the $D a$ : The $D a$ values after $1 \mathrm{~h}$ of amination with $0.25 \mathrm{M}$ TMA were almost constant at $93 \%$, regardless of the $D g$. The quaternary amine-group densities were 2.6, 3.3, 3.6, and $3.8 \mathrm{mmol}-\mathrm{TMA} / \mathrm{g}$-catalyst for $\mathrm{Dg}$ values of $100 \%, 200 \%, 300 \%$, and $400 \%$, respectively.

Next, the effect of the type of immobilized quaternary amine groups on the catalytic activity was investigated; these results are given in Figure 2. As described in Section 2.3, transesterification was conducted in batch mode,

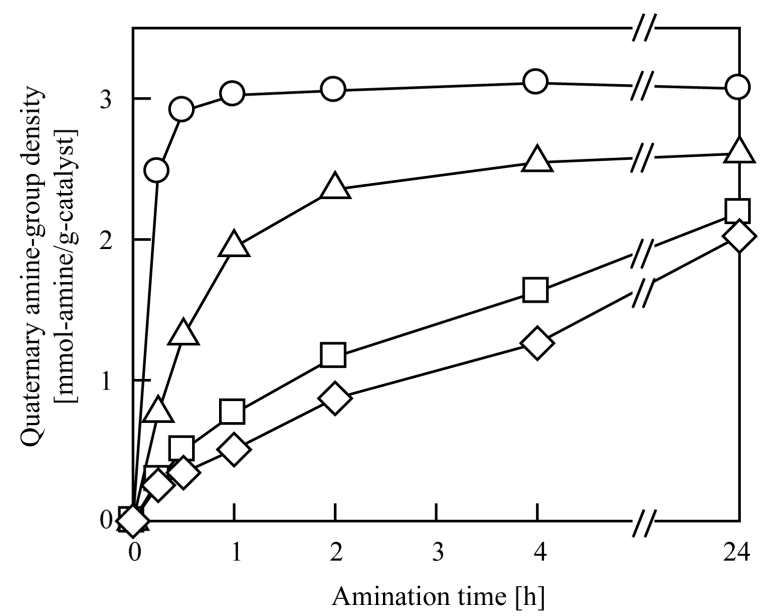

Figure 1. Effect of the type of amine reagents on the quaternary amine-group density of CMS-grafted nonwoven fabric.

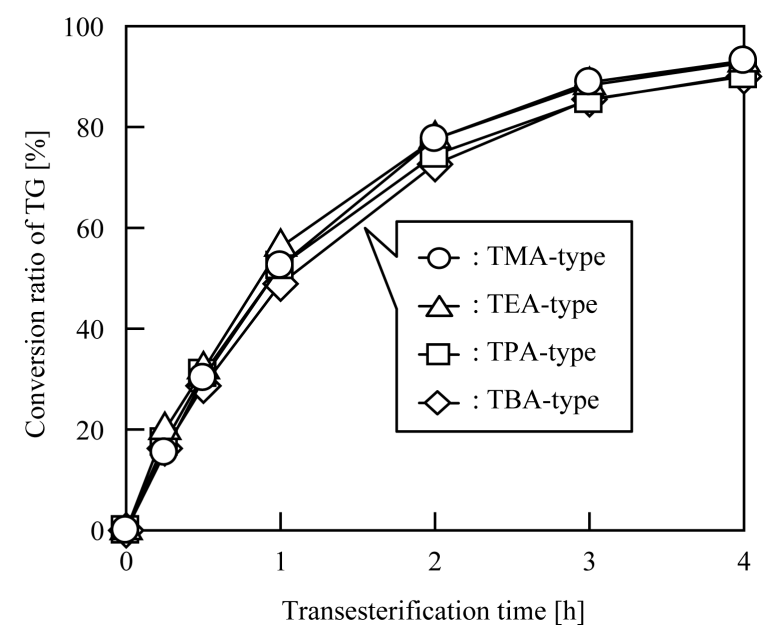

Figure 2. Effect of the type of immobilized quaternary amine group on the catalytic activity. 
and the molar quantity of quaternary amine groups of each catalyst in the reaction mixture was fixed at 0.8 mmol. As is evident from Figure 2, each catalyst exhibited similar catalytic activity toward BDF production regardless of the type of quaternary amine groups, and hence steric bulk, and transesterification proceeded with all the grafted fibrous catalysts. The conversion ratios of TG using TMA-, TEA-, TPA-, and TBA-type fibrous catalysts were $93 \%, 93 \%, 90 \%$, and $90 \%$, respectively, after $4 \mathrm{~h}$ of transesterification. The similarity of the transesterification speeds is attributed to the function of the quaternary amine groups, i.e., as a scaffold to immobilize the $\mathrm{OH}^{-}$ions that are the actual catalytic elements; therefore, the quaternary amine groups were not directly involved in transesterification. From the results of the amination of CMS-grafted nonwoven fabric and the effect of the type of quaternary amine groups on the catalytic activity, we concluded that TMA is the optimal quaternary amine group for the grafted fibrous catalyst because it is the smallest trialkylamine and thus could easily be immobilized onto the CMS-graft chains. The order of reactivity of the trialkylamines for the amination of CMSgraft chains was TMA $>>$ TEA $>$ TPA $>$ TBA.

\subsection{Effect of Catalyst Conditions on Catalytic Performance}

The yield and rate of BDF production were greatly dependent on the catalyst conditions such as the $D g$ value, the shape and the fiber diameter of the grafted fibrous catalyst, and the transesterification conditions including the molar quantity of quaternary amine groups in the reaction mixture, transesterification temperature, molar ratio of TG to EtOH in the reaction mixture, type of alcohol, and so on. In this section, the effects of the catalyst conditions on catalytic performance were investigated.

First, the effects of the $D g$ value of the grafted fibrous catalyst on the catalytic activity and yield were investigated. In this experiment, the $D g$ of the grafted fibrous catalyst was controlled within a range up to $400 \%$, and the molar quantity of TMA in the reaction mixture was $0.8 \mathrm{mmol}$ per test. The other conditions were the same as the typical conditions described in Section 2.3. Regardless of the $D g$ value, each catalyst exhibited almost the same catalytic activity toward BDF production, except in the case of the grafted fibrous catalysts with $80 \% \mathrm{Dg}$ or less. The TG conversion ratios using catalysts with $80 \%, 170 \%, 200 \%, 300 \%$, and $400 \% D g$ reached $70 \%$, $87 \%, 91 \%, 93 \%$, and $89 \%$, respectively, after $4 \mathrm{~h}$ of transesterification. The decline in the transesterification efficiency of grafted fibrous catalyst with less than $80 \% \mathrm{Dg}$ is attributed to the large volumes of these catalysts, which are sterically bulky, required to introduce $0.8 \mathrm{mmol}$ of TMA into the reaction system. As a result, part of the grafted fibrous catalyst was not immersed in the reaction mixture during transesterification. Based on these results, we concluded that the optimal $D g$ value of the grafted fibrous catalyst to achieve effective transesterification was greater than $170 \%$. The TMA group density of $170 \% \mathrm{Dg}$ is $3.1 \mathrm{mmol}-\mathrm{TMA} / \mathrm{g}$-catalyst.

The fiber diameter of the grafted fibrous catalyst is also an important factor that affects the catalytic activity; accordingly, the relationship between the fiber diameter of the grafted fibrous catalyst and catalytic activity was examined. In this test, four types of grafted fibrous catalysts with different fiber diameters (i.e., 26, 39, 47, and $67 \mu \mathrm{m})$ were used. The grafted fibrous catalysts with different fiber diameters were prepared by controlling the fiber diameter of the nonwoven fabric to be $13,19,24$, and $34 \mu \mathrm{m}$. The $\mathrm{Dg}$ and quaternary amine-group density of all grafted fibrous catalysts were fixed at approximately $300 \%$ and 3.6 mmol-TMA/g-catalyst, and the other conditions were the same as the typical conditions described in Section 2.3. Each catalyst exhibited almost the same catalytic activity toward BDF production regardless of the fiber diameter of the grafted fibrous catalyst, although the fiber diameter of the trunk polymer had a significant influence on the grafting efficiency. During emulsion grafting, trunk polymers with finer fibers had a larger specific surface area, which increased the speed and efficiency of the graft polymerization. A similar result was reported by Basuki et al. [27]. In contrast, during transesterification, the molar quantity of TMA in the reaction mixture was more important than the specific surface area because the reactants could be easily and immediately transported to the functional groups by the convective flow of the reaction mixture. Based on these results, the fiber diameter of the grafted fibrous catalyst did not significantly influence the catalytic activity. The conversion ratios of TG using the catalysts with fiber diameters of $26,39,47$, and $67 \mu \mathrm{m}$ were $93 \%, 92 \%, 88 \%$, and $90 \%$, respectively, after $4 \mathrm{~h}$ of transesterification.

\subsection{Effect of Transesterification Conditions on Catalytic Performance}

The effect of the molar quantity of quaternary amine group on the transesterification rate was investigated; these results are shown in Figure 3. In this experiment, the molar quantities of quaternary amine groups varied from 0.1 to $0.8 \mathrm{mmol}$ TMA, and the other conditions were the same as the typical conditions described in Section 2.3. 
As expected, the transesterification rate increased with increasing molar quantity of TMA in the reaction mixture, and the conversion ratios of TG using $0.1,0.2,0.4$, and $0.8 \mathrm{mmol}$ TMA reached $27 \%$, 48\%, 76\%, and 93\%, respectively, after $4 \mathrm{~h}$ of transesterification. In this experiment, we concluded that the optimal molar quantity of quaternary amine groups (i.e., TMA) to achieve effective transesterification was $0.8 \mathrm{mmol}$ per test.

Furthermore, the effect of the transesterification temperature on the transesterification rate of the grafted fibrous catalyst was studied in detail, and the results are shown in Figure 4(a). For comparison, the results for commercial granular anion exchange resin are provided in Figure 4(b). DIAION PA306S (Mitsubishi Chemical Co., Ltd., Tokyo, Japan) was used as the commercial granular anion exchange resin with TMA quaternary amine groups at a density of $4.2 \mathrm{mmol}-\mathrm{TMA} / \mathrm{g}$-dry resin with a particle diameter of 150 to $425 \mu \mathrm{m}$. In this experiment, the transesterification temperature was controlled within the range of $20^{\circ} \mathrm{C}$ to $80^{\circ} \mathrm{C}$ and TMA-type grafted fibrous catalyst was used. The other conditions were the same as the typical conditions described in Section 2.3. As indicated in Figure 4, although both the grafted fibrous catalyst and commercial granular resin exhibited good catalytic activities at all investigated transesterification temperatures, the transesterification rate of the grafted fibrous catalyst was faster than that of DIAION PA306S at all temperatures. As expected, higher transesterification temperatures led to faster transesterification rates for both catalysts. In particular, the transesterification was remarkably accelerated at high temperatures for the grafted fibrous catalyst. As seen in Figure 4(a), in the case of the grafted fibrous catalyst, about $3 \mathrm{~h}$ of transesterification was required to convert $90 \%$ of the initial TG into

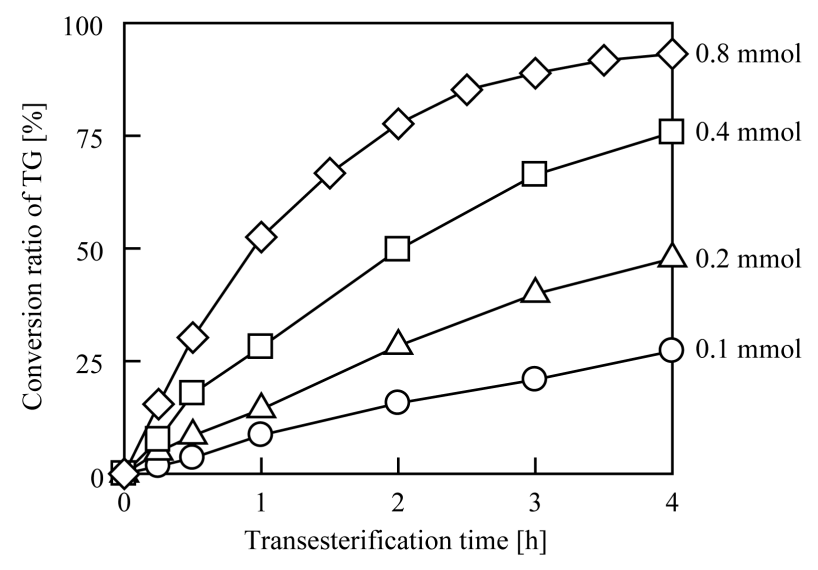

Figure 3. Effect of molar quantity of quaternary amine groups on transesterification speed.

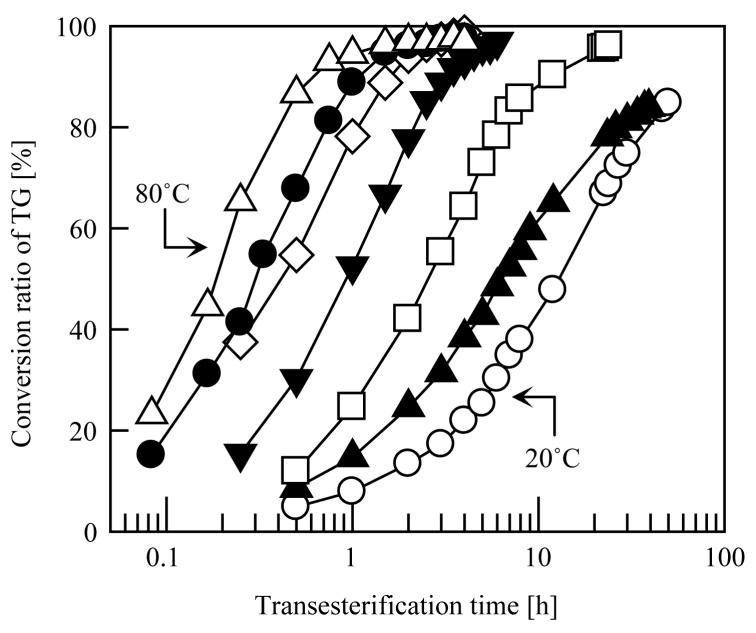

(a)

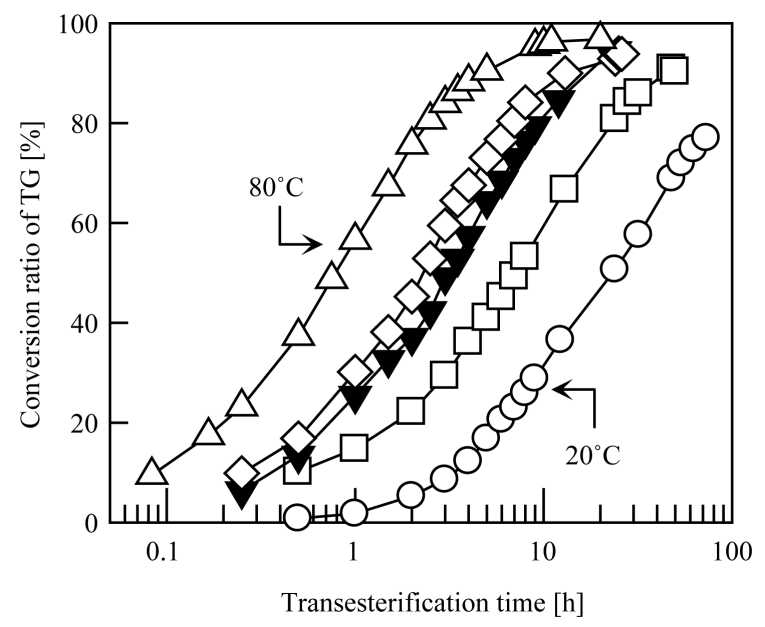

(b)

Figure 4. Effect of transesterification temperature on transesterification speed. Catalyst: (a) grafted fibrous catalyst and (b) commercial granular resin. Transesterification temperature: $\bigcirc, 20^{\circ} \mathrm{C} ; \boldsymbol{\Delta}, 30^{\circ} \mathrm{C} ; \square, 40^{\circ} \mathrm{C} ; \boldsymbol{\nabla}, 50^{\circ} \mathrm{C} ; \diamond, 60^{\circ} \mathrm{C} ; \boldsymbol{Q}, 70^{\circ} \mathrm{C}$; $\triangle, 80^{\circ} \mathrm{C}$. 
$\mathrm{BDF}$ at $50^{\circ} \mathrm{C}$. In contrast, at $80^{\circ} \mathrm{C}$, the required time to achieve a conversion ratio of $90 \%$ was only 40 min, which is less than one fourth the time required at $50^{\circ} \mathrm{C}$. Based on these results, higher reaction temperatures resulted in more effective and efficient transesterification and, therefore, the grafted fibrous catalyst could dramatically reduce the time required for BDF production than the granular resin.

The ratio of TG to alcohol in the reaction mixture is also known to be a controlling factor for BDF production. Therefore, the effects of the molar ratio of TG to EtOH in the reaction mixture on the transesterification yield and rate were investigated; the results are given in Figure 5. In this experiment, the molar ratios of TG to EtOH were adjusted to $1: 3,1: 10,1: 25,1: 50,1: 100,1: 200$, and 1:500, which corresponded to TG/EtOH volume ratios of 1:0.2, 1:0.6, 1:1.5, 1:3, 1:6, 1:12, and 1:30, respectively. The other conditions were the same as the typical conditions described in Section 2.3. As denoted in Figure 5, the molar ratio of TG to EtOH had a significant influence on the transesterification yield and rate: Transesterification was accelerated in the presence of excess $\mathrm{EtOH}$. The transesterification times required to convert $90 \%$ of the initial TG into BDF were 4.47, 3.18, 2.09, 1.51 and $1.56 \mathrm{~h}$ for TG/EtOH molar ratios of 1:25, 1:50, 1:100, 1:200 and 1:500, respectively. TG/EtOH molar ratios of $1: 3$ and $1: 10$, the conversion ratio did not reach $90 \%$ even after $50 \mathrm{~h}$. There are two potential reasons for these phenomena: One is that the $\mathrm{EtOH}$ in the reaction mixture was vaporized by heating during transesterification, and the other is that the relatively low volume proportion of EtOH to TG in the reaction mixture resulted in heterogeneous dispersion of the EtOH. The TG/EtOH molar ratios of 1:3 and 1:10 were corresponded to the TG/EtOH volume ratios of 1:0.2 and 1:0.6, respectively. In contrast, when the percentage of EtOH in the reaction mixture was significantly high, as at a TG/EtOH molar ratio of 1:500, EtOH diluted the reaction mixture and thereby hindered the rate of transesterification. Based on these results, the optimal molar ratio of TG and $\mathrm{EtOH}$ in the reaction mixture for the fastest BDF production was 1:200.

Finally, the effects of the type of alcohol on the transesterification yield and speed were investigated. In this test, nine primary alcohols with different linear alkyl chain lengths, i.e., 1 to 12 carbon atoms, were used; these were methanol (C1), EtOH (C2), 1-propanol (C3), 1-butanol (C4), 1-pentanol (C5), 1-hexanol (C6), 1-octanol (C8), 1-decanol (C10), and 1-dodecanol (C12). The molar ratio of TG to primary alkyl alcohol was fixed at 1:50, and the other conditions were the same as the typical conditions described in Section 2.3. Figure 6 shows HPLC chromatograms of the BDF samples produced from 30 min of transesterification of triolein with each alcohol, and the conversion ratio of TG with each alcohol is plotted versus the transesterification time in Figure 7. As shown in Figure 6, BDF was produced regardless of the primary alkyl alcohol used; therefore, the grafted fibrous catalyst is applicable to transesterification of TG with a variety of alcohols. Furthermore, the peaks corresponding to BDF gradually shifted to longer retention times as the alkyl chain length of the primary alkyl alcohol increased; this is attributed to the differences in the structures (and hydrophobicity) of the produced BDF, and

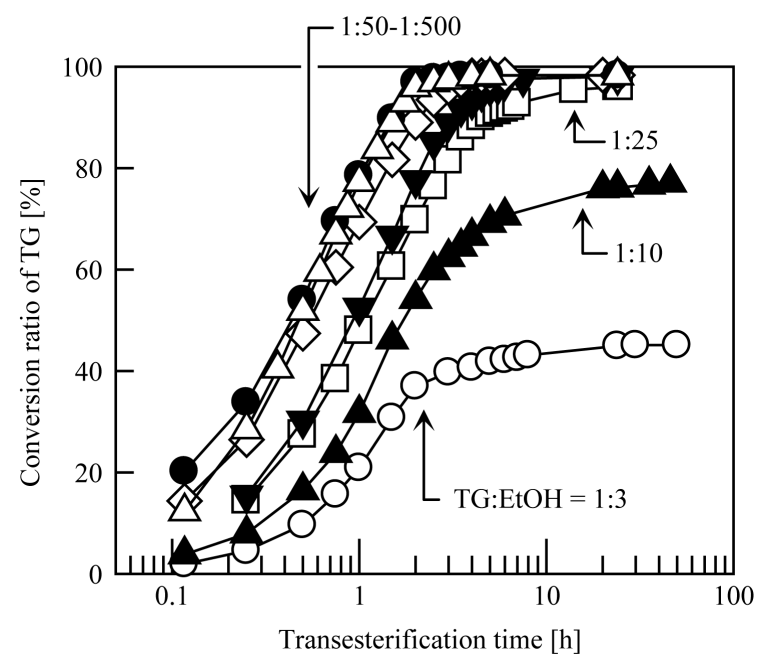

Figure 5. Effects of molar ratio of TG to EtOH in reaction mixture on transesterification yield and speed. Molar ratio of TG to EtOH: $\bigcirc, 1: 3 ; \boldsymbol{\Delta}, 1: 10 ; \square, 1: 25 ; \boldsymbol{\nabla}$ $1: 50 ; \diamond, 1: 100 ; \bigcirc, 1: 200 ; \triangle, 1: 500$. 

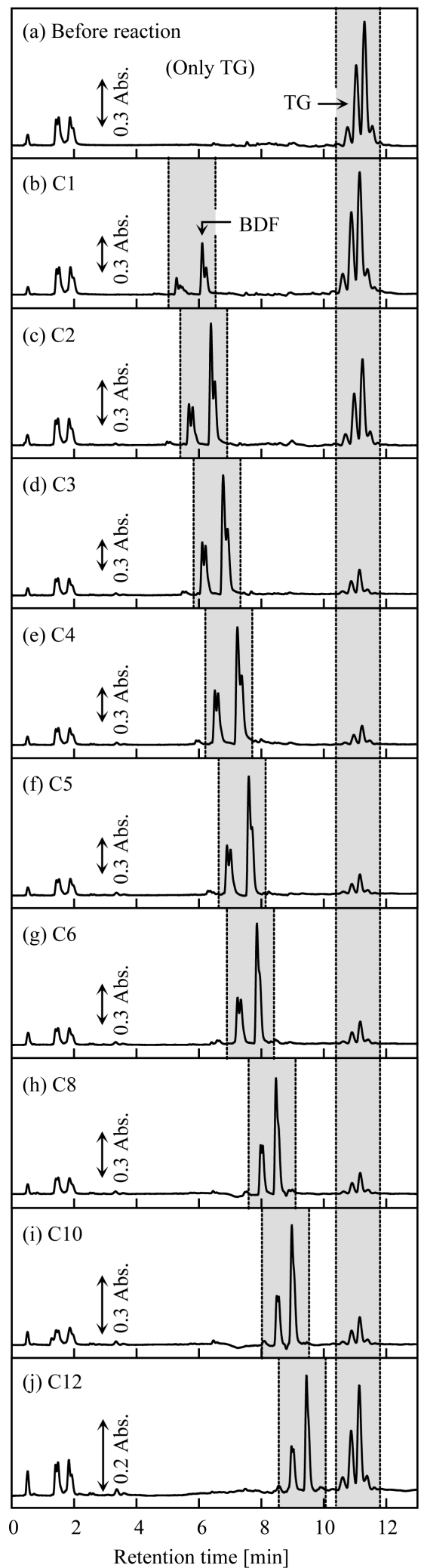

Figure 6. HPLC chromatograms of BDF samples produced by transesterification of triolein with different types of alcohol. Transesterification time: $30 \mathrm{~min}$. 


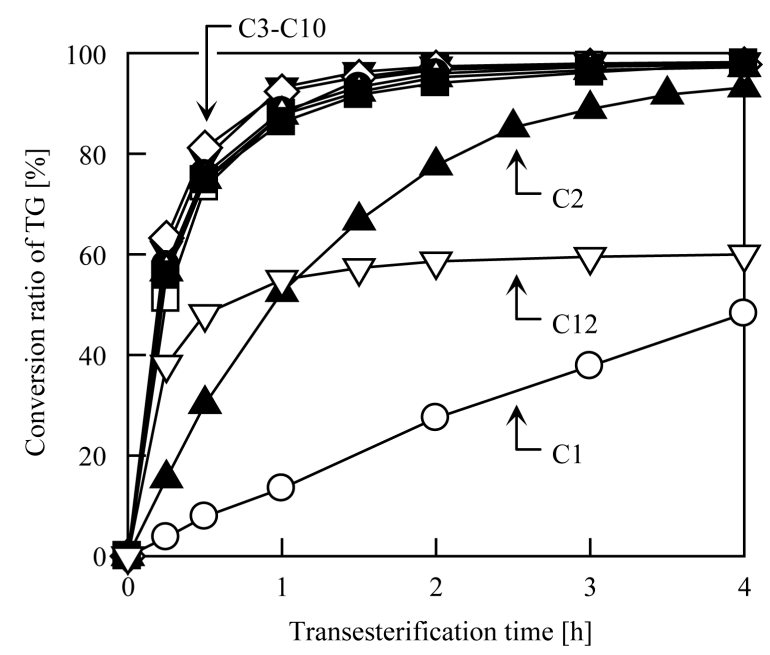

Figure 7. Effects of the type of alcohol on transesterification yield and speed. Alcohol: $\mathrm{O}, \mathrm{C} 1 ; \boldsymbol{\Delta}, \mathrm{C} 2 ; \square$, C3; $\nabla$, C4; $\diamond, \mathrm{C} 5 ; \bigcirc, \mathrm{C} 6 ; \triangle, \mathrm{C} 8 ; \mathbf{\square}, \mathrm{C} 10 ; \nabla, \mathrm{C} 12$.

demonstrated that differed BDFs were produced from different types of alcohol. As can be seen from Figure 7, the yield and speed of BDF production varied depending on the type of primary alkyl alcohol: The conversion ratios of TG using C1, C2, C3, C4, C5, C6, C8, C10, and C12 were 8\%, 30\%, 73\%, 79\%, 81\%, 76\%, 75\%, 74\%, and $48 \%$, respectively, after $30 \mathrm{~min}$ of transesterification. These results indicate that transesterification is acelerated when more hydrophobic (i.e., lipophilic) primary alkyl alcohols were used. However, as is evident from the results of using C12, very hydrophobic alcohols resulted in slower transesterification than primary alkyl alcohols with moderate hydrophobicity, such as C4, C5, and C6. Furthermore, transesterification using C12 slowed significantly at the conversion ratio of about $55 \%$ during the first $1 \mathrm{~h}$; afterward, transesterification progressed gradually to reach a conversion ratio of $64 \%$ after $24 \mathrm{~h}$. This decline in transesterification yield is attributed to the primary alkyl alcohol being too hydrophobic to react with the $\mathrm{OH}^{-}$ions, which are highly hydrophilic, although highly hydrophobic alcohols are highly miscible with TG. In contrast, when primary alkyl alcohols with shorter alkyl chains, such as C1, were used, phase separation occurred before and after transesterification, even if a cosolvent such as decane was added to improve the uniformity of the reaction solution. This phase separation is attributed to the immiscibility of TG and C1. As a result, as shown in Figure 7, the trancesterification speed using methanol was very slow, because the reaction occurred only at the interface between the TG and C1 layer. The conversion ratio of TG using C1 reached 96\% after $24 \mathrm{~h}$ of transesterification. To increase the miscibility of the two compounds, Tang et al. suggested that higher pressure and temperature are needed [28]. Based on the above results, the structure of BDF and transesterification speed could be controlled to some extent by the type of alcohol used. In this study, the primary alkyl alcohol that enabled the fastest production of BDF was C5, and the order of transesterification speed was $\mathrm{C} 1<<\mathrm{C} 2<<\mathrm{C} 3<\mathrm{C} 4<\mathrm{C} 5>$ C6 $>$ C8 $>$ C10 $>>$ C12.

\subsection{BDF Production from Vegetable Oils and Animal Fat}

From the above experimental results, it was elucidated that the grafted fibrous catalyst shows good activity for BDF production from TG with an alcohol. To enable practical use of the grafted fibrous catalyst, it must adapt to a wide variety of feed oils such as natural vegetable oils, animal fats, and waste oils, which contain different types of TGs [29] [30], in addition to the reagent-grade triolein used in this study. Thus, the effect of the type of natural oil on the transesterification yield and speed was investigated. In this test, four types of vegetable oils, i.e., linseed oil, safflower oil, rapeseed oil, and palm oil, and one type of animal fat, i.e., beef tallow, were used. EtOH was used as the primary alkyl alcohol, and the reaction mixture was composed of $1.4 \mathrm{~g}$ of natural oils, 3.6 $\mathrm{g}$ of EtOH, and $5.0 \mathrm{~g}$ of decane. The other conditions were the same as the typical conditions described in Section 2.3. Figure 8 shows the HPLC chromatograms of the BDF samples produced using the different types of natural oils before and after $4 \mathrm{~h}$ of transesterification. In the chromatograms of the natural oils, many peaks 


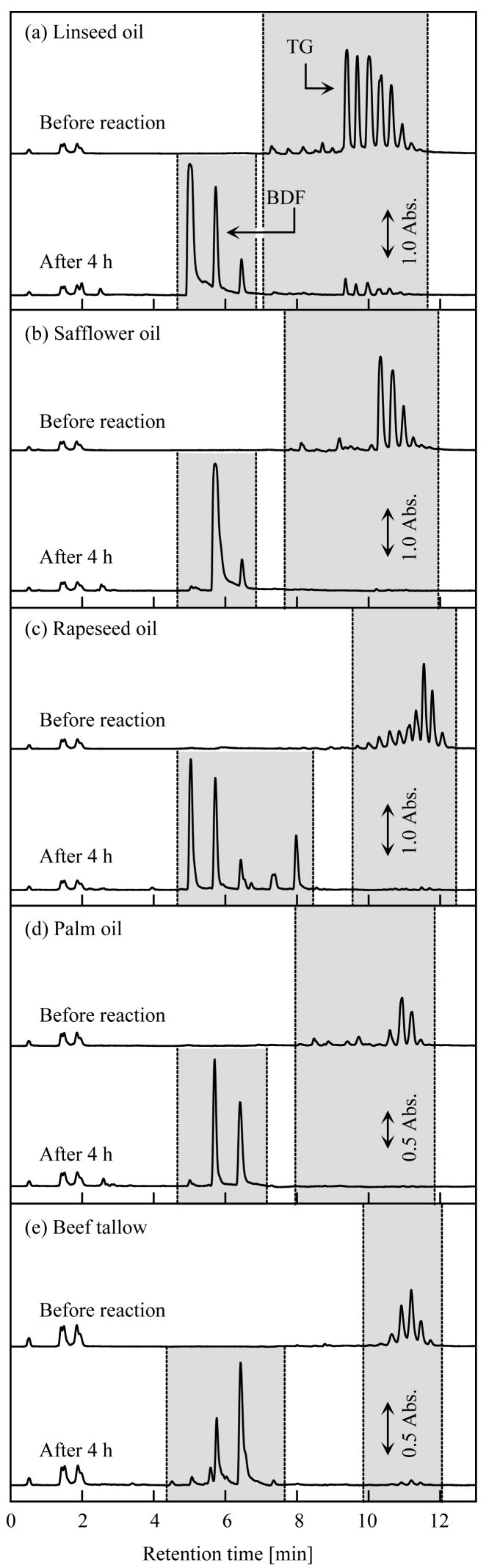

Figure 8. BDF production from vegetable oils and animal fat using grafted fibrous catalyst. 
derived from TG, which was contained in the natural oils, were observed before transesterification, unlike in the chromatogram of reagent-grade triolein. Although the transesterification speed differed according to the type of natural oil, the conversion ratios for all the natural oils were greater than $90 \%$ after $4 \mathrm{~h}$ of transesterification. The conversion ratios for linseed oil, safflower oil, rapeseed oil, palm oil, and beef tallow were 92\%, 98\%, 97\%, $97 \%$, and $93 \%$, respectively, after $4 \mathrm{~h}$ of transesterification. Based on these results, it is evident that the grafted fibrous catalyst is applicable for BDF production from many types of natural oils.

\subsection{Repeated-Use Stability and Regeneration of Grafted Fibrous Catalyst}

Repeated-use stability and ease of regeneration are important aspects of the grafted fibrous catalyst for the production of large amounts of BDF and extended use of the catalyst. Firstly, the repeated-use stability of the grafted fibrous catalyst was evaluated by repeated transesterification of triolein with $\mathrm{EtOH}$, and the immutability of the catalytic activity for each transesterification experiment was monitored. In this test, the grafted fibrous catalyst was only washed with EtOH after each transesterification. The molar ratio of TG to EtOH was fixed at $1: 200$, and the other conditions were the same as the typical conditions described in Section 2.3. As shown in Figure 9, the catalytic activity of the grafted fibrous catalyst gradually decreased with increasing number of transesterification reactions. There are two main reasons for this decay of catalytic activity: One is the removal of $\mathrm{OH}^{-}$ions from the grafted fibrous catalyst, and the other is contamination of the quaternary amine groups by oleate ions, which are catalytically inactive. Oleate ions are generated during the transesterification process by a direct ion-exchange reaction of the $\mathrm{OH}^{-}$ions, which were immobilized onto the graft chains, with the oleic acid group of triolein, diolein, or monoolein. In the presence of organic acid ions, the counterion of the quaternary amine group that was introduced into the graft chain is easily replaced with an organic acid ion instead of a $\mathrm{OH}^{-}$ ion. Additionally, rate of decrease of the catalytic activity was not steady: A rapid decrease was observed after the $8^{\text {th }}$ and subsequent transesterifications. The transesterification times required to convert $90 \%$ of the initial TG into BDF were 0.99, 1.00, 1.04, 1.10, 1.18, 1.32, 1.44, 1.97, 2.90 and $12.00 \mathrm{~h}$ for the first ten reactions. At the $11^{\text {th }}$ reaction, the catalytic activity of the grafted fibrous catalyst was almost negligible; thus, the conversion ratio only reached $9 \%$, even after $24 \mathrm{~h}$ of transesterification.

Next, the deactivated grafted fibrous catalyst was regenerated, according to the procedure described by Shibasaki-kitakawa et al. [12]. The regeneration process consists of the following three steps: 1) Washing with 0.25 $\mathrm{M}$ citric acid solution (solvent: EtOH) to desorb the organic acid ions that cover the active sites, i.e., the quaternary amine groups, of the grafted fibrous catalyst, 2) regenerating with $1 \mathrm{M} \mathrm{NaOH}$ aqueous solution to replace the citric acid ions, which formed ionic bonds with the quaternary amine groups, with $\mathrm{OH}^{-}$ions and washing with deionized water, and 3) washing with $\mathrm{EtOH}$ to restore the initial swelled condition. When the deactivated grafted fibrous catalyst was treated with the first and third steps and only the third step, the catalytic activities of

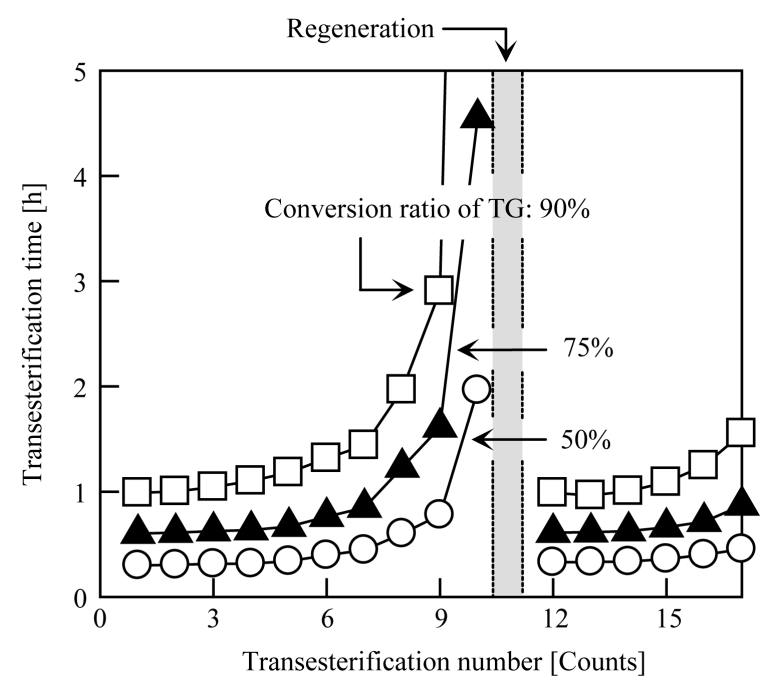

Figure 9. Repeated-use stability and regeneration of grafted fibrous catalyst. 
each grafted fibrous catalyst did not recover their original state. The conversion ratios of each grafted fibrous catalyst were almost zero, even after $24 \mathrm{~h}$ of transesterification. When the deactivated grafted fibrous catalyst was treated with the second and third steps, the catalytic activity of the grafted fibrous catalyst partially recovered: The conversion ratio after $1 \mathrm{~h}$ of transesterification was $25 \%$, which is equivalent to about one-fourth of the original ratio ( $90 \%$ after $1 \mathrm{~h}$ of transesterification). Also, the transesterification reaction almost stopped after $4 \mathrm{~h}$, and the conversion ratio after $4 \mathrm{~h}$ was about $43 \%$. However, when the deactivated grafted fibrous catalyst was treated with all three steps, the catalytic activity of the grafted fibrous catalyst almost completely recovered to its original state:

The transesterification times required to convert $90 \%$ of the initial TG into BDF were 0.99 and $0.99 \mathrm{~h}$ for the $1^{\text {st }}$ and $12^{\text {th }}$ reactions, respectively. The conversion ratio and transesterification speed of the regenerated grafted fibrous catalyst almost overlapped with those of the unused grafted fibrous catalyst. As shown in Figure 9, the grafted fibrous catalyst could be used repeatedly, although the catalytic activity of the regenerated grafted fibrous catalyst gradually decreased with increasing number of transesterification reactions, as was the case with the unused grafted fibrous catalyst. Additionally, the regenerated grafted fibrous catalyst did not suffer significant physical or chemical damage, even after several transesterification-regeneration cycles. Based on these results, it is evident that the grafted fibrous catalyst could be used up to ten times without any proper regeneration and the BDF production capacity per gram of the grafted fibrous catalyst with 3.6 mmol-TMA/g-catalyst was $70 \mathrm{~g}$ because the weight ratio of triolein to grafted fibrous catalyst in the reaction mixture was fixed at 7:1 (1.4 g/0.2 g) per test. Furthermore, it was confirmed that the three sequential regeneration processes, which involved organic acid, alkali and alcohol treatments, were required to completely recover the catalytic activity of the deactivated grafted fibrous catalyst. Also, the grafted fibrous catalysts are sufficiently stable for repeated use and sufficiently durable for long-term use.

\section{Conclusion}

TMA is the optimal quaternary amine group for the grafted fibrous catalyst from both perspectives of the ease of amination and the catalytic performance, although the various types of quaternary amine groups with different alkyl chain lengths could be introduced into the CMS-graft chains. The optimal $D g$ value of the grafted fibrous catalyst to achieve effective transesterification was greater than $170 \%$, andthe quaternary amine-group density of $170 \% \mathrm{Dg}$ is $3.1 \mathrm{mmol}-\mathrm{TMA} / \mathrm{g}$-catalyst. Additionally, the transesterification temperature, molar ratio of TG to $\mathrm{EtOH}$, and type of alcohol significantly influenced the transesterification efficiency, as did the molar quantity of quaternary amine groups in the reaction mixture. The optimal molar quantity of quaternary amine groups, transesterification temperature, molar ratio of TG and $\mathrm{EtOH}$, and primary alkyl alcohol were $0.8 \mathrm{mmol}, 80^{\circ} \mathrm{C}, 1: 200$, and 1-pentanol, respectively. The grafted fibrous catalyst could be applied to BDF production using any type of natural oil as the starting oil. The grafted fibrous catalyst could be used ten times without any proper regeneration, and the BDF production capacity per gram of the grafted fibrous catalyst was $\sim 70 \mathrm{~g}$. The deactivated grafted fibrous catalyst could be recovered to its initial state by three sequential regeneration processes, including organic acid, alkali, and alcohol treatments, without any significant loss of the catalytic activity. Based on these results, the grafted fibrous catalyst, which has sufficient catalytic activity, efficient BDF production capacity, and high repeated-use stability for practical application in BDF production, will contribute to the feasibility of environmentally friendly energy resources, reduce the dependence on petroleum, and improve global environment preservation in the near future. Furthermore, we are firmly convinced that this new type of catalyst will encourage the dissemination of radiation processing and create new possibilities for radiation research.

\section{References}

[1] US Department of Energy, Energy Information Administration (US DOE/EIA) (2001) International Energy Outlook 2001. US Department of Energy, Energy Information Administration, Washington DC.

[2] US Department of Energy, Energy Information Administration (US DOE/EIA) (2011) International Energy Outlook 2011. US Department of Energy, Energy Information Administration, Washington DC.

[3] Monlau, F., Sambusiti, C., Barakat, A., Guo, X.M., Latrille, E., Trably, E., Steyer, J.-P. and Carrere, H. (2012) Predictive Models of Biohydrogen and Biomethane Production Based on the Compositional and Structural Features of Lignocellulosic Materials. Environmental Science \& Technology, 46, 12217-12225. http://dx.doi.org/10.1021/es303132t

[4] Li, Y., Zhang, R., Liu, X., Chen, C., Xiao, X., Feng, L., He, Y. and Liu, G. (2013) Evaluating Methane Production 
from Anaerobic Mono- and Co-Digestion of Kitchen Waste, Corn Stover, and Chicken Manure. Energy \& Fuels, 27, 2085-2091. http://dx.doi.org/10.1021/ef400117f

[5] Yangcheng, H., Jiang, H., Blanco, M. and Jane, J.-L. (2013) Characterization of Normal and Waxy Corn Starch for Bioethanol Production. Journal of Agricultural and Food Chemistry, 61, 379-386. http://dx.doi.org/10.1021/jf305100n

[6] Limayem, A. and Ricke, S.C. (2012) Lignocellulosic Biomass for Bioethanol Production: Current Perspectives, Potential Issues and Future Prospects. Progress in Energy and Combustion Science, 38, 449-467. http://dx.doi.org/10.1016/j.pecs.2012.03.002

[7] Tran, H.-L., Ryu, Y.-J., Seong, D.H., Lim, S.-M. and Lee, C.-G. (2013) An Effective Acid Catalyst for Biodiesel Production from Impure Raw Feedstocks. Biotechnology and Bioprocess Engineering, 18, 242-247. http://dx.doi.org/10.1007/s12257-012-0674-1

[8] Sagiroglu, A., Ozcan, H.M., Isbilir, S.S., Paluzar, H. and Toprakkiran, N.M. (2013) Alkali Catalysis of Different Vegetable Oils for Comparisons of Their Biodiesel Productivity. Journal of Sustainable Bioenergy Systems, 3, 79-85. http://dx.doi.org/10.4236/jsbs.2013.31011

[9] Mata, T.M., Sousa, I.R.B.G., Vieira, S.S. and Caetano, N.S. (2012) Biodiesel Production from Corn Oil via Enzymatic Catalysis with Ethanol. Energy \& Fuels, 26, 3034-3041. http://dx.doi.org/10.1021/ef300319f

[10] Babajide, O., Musyoka, N., Petrik, L. and Ameer, F. (2012) Novel Zeolite Na-X Synthesized from Fly Ash as a Heterogeneous Catalyst in Biodiesel Production. Catalysis Today, 190, 54-60. http://dx.doi.org/10.1016/j.cattod.2012.04.044

[11] Ilham, Z. and Saka, S. (2012) Optimization of Supercritical Dimethyl Carbonate Method for Biodiesel Production. Fuel, 97, 670-677. http://dx.doi.org/10.1016/i.fuel.2012.02.066

[12] Shibasaki-Kitakawa, N., Honda, H., Kuribayashi, H., Toda, T., Fukumura, T. and Yonemoto, T. (2007) Biodiesel Production Using Anionic Ion-Exchange Resin as Heterogeneous Catalyst. Bioresource Technology, 98, 416-421. http://dx.doi.org/10.1016/j.biortech.2005.12.010

[13] Tsuji, T., Kubo, M., Shibasaki-Kitakawa, N. and Yonemoto, T. (2009) Is Excess Methanol Addition Required to Drive Transesterification of Triglyceride toward Complete Conversion? Energy \& Fuels, 23, 6163-6167. http://dx.doi.org/10.1021/ef900622d

[14] Demirbas, A. (2008) Comparison of Transesterification Methods for Production of Biodiesel from Vegetable Oils and Fats. Energy Conversion and Management, 49, 125-130. http://dx.doi.org/10.1016/j.enconman.2007.05.002

[15] Helwani, Z., Othman, M.R., Aziz, N., Kim, J. and Fernando, W.J.N. (2009) Solid Heterogeneous Catalysts for Transesterification of Triglycerides with Methanol: A Review. Applied Catalysis A: General, 363, 1-10. http://dx.doi.org/10.1016/j.apcata.2009.05.021

[16] Zhou, D., Zhang, S., Fu, H. and Chen, J. (2012) Liquefaction of Macroalgae Enteromorpha Prolifera in Sub-/Supercritical Alcohols: Direct Production of Ester Compounds. Energy \& Fuels, 26, 2342-2351. http://dx.doi.org/10.1021/ef201966w

[17] Menetrez, M.Y. (2012) An Overview of Algae Biofuel Production and Potential Environmental Impact. Environmental Science \& Technology, 46, 7073-7085. http://dx.doi.org/10.1021/es300917r

[18] Ueki, Y., Mohamed, N.H., Seko, N. and Tamada, M. (2011) Rapid Biodiesel Fuel Production Using Novel Fibrous Catalyst Synthesized by Radiation-Induced Graft Polymerization. International Journal of Organic Chemistry, 1, 20-25. http://dx.doi.org/10.4236/ijoc.2011.12004

[19] Madrid, J.F., Ueki, Y. and Seko, N. (2013) Abaca/Polyester Nonwoven Fabric Functionalization for Metal Ion Adsorbent Synthesis via Electron Beam-Induced Emulsion Grafting. Radiation Physics and Chemistry, 90, 104-110. http://dx.doi.org/10.1016/j.radphyschem.2013.05.004

[20] Ueki, Y., Dafader, N.C., Hoshina, H., Seko, N. and Tamada, M. (2012) Study and Optimization on Graft Polymerization under Normal Pressure and Air Atmospheric Conditions, and Its Application to Metal Adsorbent. Radiation Physics and Chemistry, 81, 889-898. http://dx.doi.org/10.1016/j.radphyschem.2012.02.031

[21] Iwanade, A., Kasai, N., Hoshina, H., Ueki, Y., Saiki, S. and Seko, N. (2012) Hybrid Grafted Ion Exchanger for Decontamination of Radioactive Cesium in Fukushima Prefecture and Other Contaminated Areas. Journal of Radioanalytical and Nuclear Chemistry, 293, 703-709. http://dx.doi.org/10.1007/s10967-012-1721-2

[22] Hoshina, H., Kasai, N., Shibata, T., Aketagawa, Y., Takahashi, M., Yoshii, A., Tsunoda, Y. and Seko, N. (2012) Synthesis of Arsenic Graft Adsorbents in Pilot Scale. Radiation Physics and Chemistry, 81, 1033-1035. http://dx.doi.org/10.1016/j.radphyschem.2012.02.018

[23] Hoshina, H., Seko, N., Ueki, Y., Iyatomi, Y. and Tamada, M. (2010) Evaluation of Graft Adsorbent with N-MethylD-Glucamine for Boron Removal from Groundwater. Journal of Ion Exchange, 21, 153-156.

[24] Seko, N., Hoshina, H., Kasai, N., Ueki, Y., Tamada, M., Kiryu, T., Tanaka, K. and Takahashi, M. (2010) Novel Sys- 
tem for Recovering Scandium from Hot Spring Water with Fibrous Graft Adsorbent. Journal of Ion Exchange, 21, 117-122.

[25] Seko, N., Katakai, A., Hasegawa, S., Tamada, M., Kasai, N., Takeda, H., Sugo, T. and Saito, K. (2003) Aquaculture of Uranium in Seawater by a Fabric-Adsorbent Submerged System. Nuclear Technology, 144, 274-278.

[26] Holčapek, M., Jandera, P., Fischer, J. and Prokeš, B. (1999) Analytical Monitoring of the Production of Biodiesel by High-Performance Liquid Chromatography with Various Detection Methods. Journal of Chromatography A, 858, 13-31. http://dx.doi.org/10.1016/S0021-9673(99)00790-6

[27] Basuki, F., Seko, N. and Tamada, M. (2010) Recovery of Scandium with Phosphoric Chelating Adsorbent Prepared by Direct Radiation Graft Polymerization. Journal of Ion Exchange, 21, 127-130.

[28] Tang, Z., Du, Z., Min, E., Gao, L., Jiang, T. and Han, B. (2006) Phase Equilibria of Methanol-Triolein System at Elevated Temperature and Pressure. Fluid Phase Equilibria, 239, 8-11. http://dx.doi.org/10.1016/j.fluid.2005.10.010

[29] Gunstone, F.D., Hamilton, R.J., Padley, F.B. and Qureshi, M.I. (1965) Glyceride Studies. V. The Distribution of Unsaturated Acyl Groups in Vegetable Triglycerides. Journal of the American Oil Chemists Society, 42, 965-970. http://dx.doi.org/10.1007/BF02632456

[30] Ramos, M.J., Fernández, C.M., Casas, A., Rodríguez, L. and Pérez, Á. (2009) Influence of Fatty Acid Composition of Raw Materials on Biodiesel Properties. Bioresource Technology, 100, 261-268. http://dx.doi.org/10.1016/j.biortech.2008.06.039 
Scientific Research Publishing (SCIRP) is one of the largest Open Access journal publishers. It is currently publishing more than 200 open access, online, peer-reviewed journals covering a wide range of academic disciplines. SCIRP serves the worldwide academic communities and contributes to the progress and application of science with its publication.

Other selected journals from SCIRP are listed as below. Submit your manuscript to us via either submit@scirp.org or Online Submission Portal.
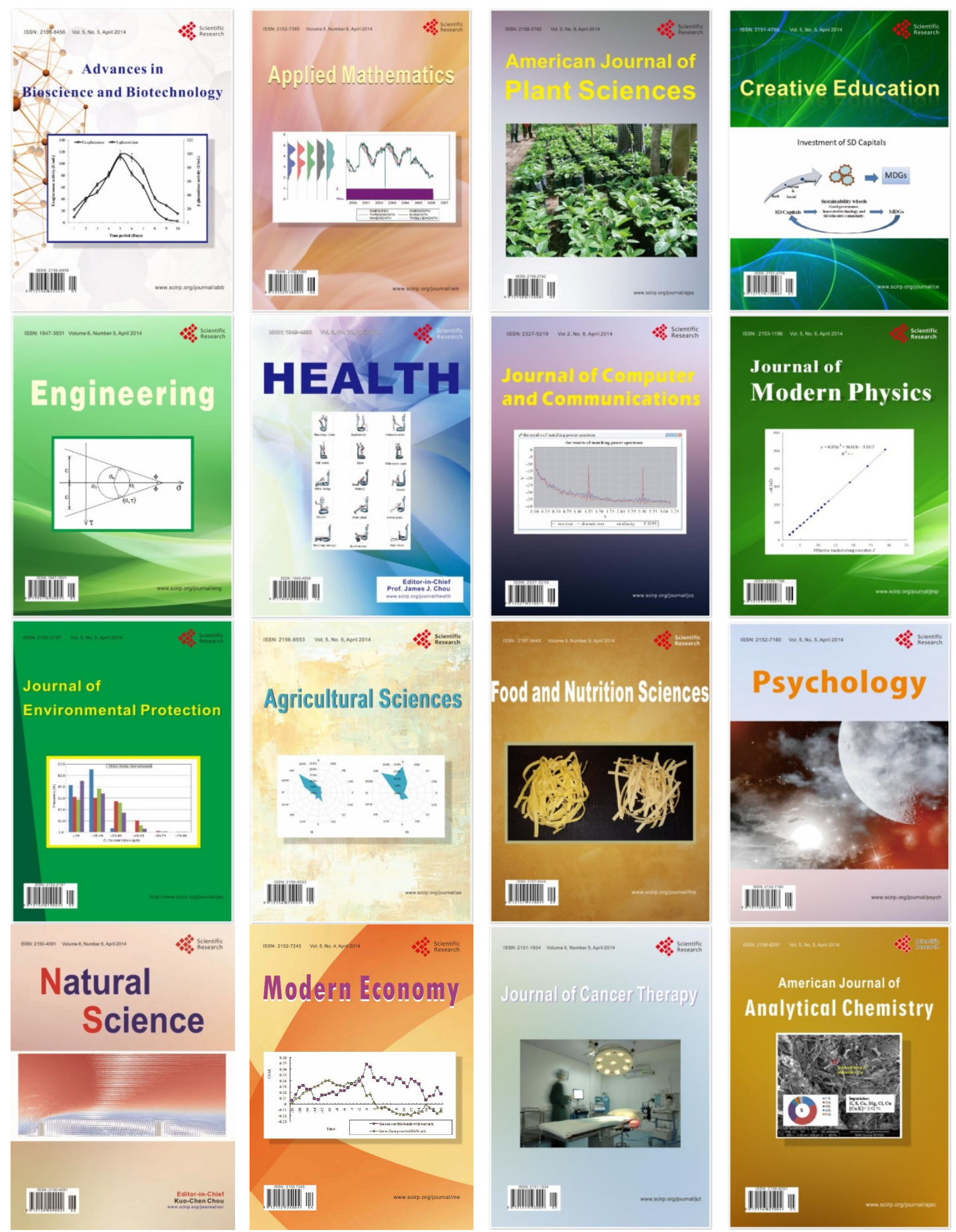